\title{
Prevalência de baixos níveis de força muscular e fatores associados em adolescentes de uma cidade do sul do Brasil
}

http://dx.doi.org/10.11606/1807-5509201900010115

\author{
Tiago Rodrigues de LIMA* \\ Gabriel Renaldo de SOUSA* \\ João Antônio Chula de CASTRO** \\ Diego Augusto Santos SILVA ${ }^{* * *}$ \\ ${ }^{*}$ Centro de \\ Ciências da Saúde, \\ Universidade Federa \\ de Santa Catarina, \\ Florianópolis, SC, \\ Brasil. \\ ${ }^{* *}$ Centro de \\ Desportos, \\ Universidade Federa \\ de Santa Catarina, \\ Florianópolis, SC \\ Brasil.
}

\section{Resumo}

A força muscular é necessária para realização de atividades diárias, sendo considerada marcador de saúde global. 0 objetivo deste estudo foi estimar a prevalência de baixos níveis de força de preensão manual (FPM) em adolescentes e verificar fatores (sociodemográficos, estilo de vida e status de peso) correlatos. A população de escolares da cidade de São José, SC, Brasil, era formada por 5.182 estudantes. Para o presente estudo de base escolar e delineamento transversal, 923 adolescentes de 14 a 19 anos de idade foram avaliados. Analisou-se a FPM por meio de dinamometria manual e protocolo proposto pela Sociedade Canadense de Fisiologia do Exercício cujo ponto de corte para baixos níveis de força é $\leq 53,0 \mathrm{kgf}$ para meninas e $\leq 83,0 \mathrm{kgf}$ para meninos. As variáveis independentes analisadas foram idade, escolaridade paterna e materna, renda familiar, atividade física, tabagismo, hábitos alimentares e status de peso. Utilizou-se teste T de Student, U de Mann-Whitney e regressão logística binária para estimar as razões de chances e intervalos de confiança de $95 \%$. A prevalência de baixos niveis de FPM foi de $59,7 \%$ nas meninas e $66,3 \%$ nos meninos. As meninas que não faziam ingestão de bebidas alcoólicas em excesso e eutróficas, e os meninos cujas mães estudaram até oito anos e de renda baixa foram os subgrupos com maiores chances de baixos niveis de força de FPM. Esforços para aumentar os níveis de FPM devem ser focados nas meninas com status do peso normal e meninos cujas mães têm menor escolaridade e renda. Futuros inquéritos com delineamento longitudinal devem ser propostos para identificar preditores dos baixos niveis de força muscular em adolescentes.

Palavras-Chave: Dinamômetro de Força Muscular; Epidemiologia; Estudos Transversais; Força da Mão; Saúde Pública.

\section{Introdução}

A força muscular é necessária para realização de tarefas diárias, laborais, recreativas e performance física, além de ser importante para a independência funcional do indivíduo ${ }^{1,2}$. Em crianças e adolescentes, a força muscular é fundamental para coordenação e progressão de habilidades motoras, sendo influenciada durante este período de transição da vida humana pela maturação biológica, que é responsável pelo amadurecimento dos órgáos sexuais e estimulação da produção de hormônios (testosterona e estrogênio) relacionados ao aumento de massa muscular e força² ${ }^{2}$.
Estudos demonstraram que a força muscular é importante componente associado à saúde, independentemente da idade ou condição clínica ${ }^{3,4}$. Em estudo longitudinal realizado com adolescentes da Suécia, verificou-se que elevados níveis de força muscular (mensurados por extensão de joelhos e preensão manual) foram associados à diminuição de risco (20-35\%) de mortalidade prematura por qualquer causa ou doenças cardiovasculares, independentemente do índice de massa corporal (IMC) ou pressão sanguínea quando adultos ${ }^{3}$. 
Diante da importância deste componente neuromuscular da aptidão física para promoção da saúde, outros elementos sáo estudados no intuito de fornecer informaçóes acerca de fatores correlatos a baixos níveis de força, como variáveis sociodemográficas ${ }^{5-7}$. Entre as variáveis sociodemográficas que se associam a baixos escores de força em adolescentes tem-se a menor faixa etária, em que jovens pré-púberes apresentaram menores níveis de força em relação aos púberes; o sexo, cujas concentraçóes plasmáticas dos principais hormônios metabólicos (testosterona e $\mathrm{GH}$ ) sáo menores nas meninas em comparação aos meninos, o que resulta em menor massa muscular e menores níveis de força; o baixo nível econômico, que pode refletir em menores possibilidades de acesso às atividades físicas no período do lazer que, por sua vez, resulta em maior peso corporal e menor desenvolvimento da força muscular ${ }^{4,5,7,8}$.

Variáveis do estilo de vida também se associam a baixos níveis de força, como o consumo de álcool, uma vez que a sua ingestão pode influenciar negativamente na síntese protéica, levando a diminuição do metabolismo celular, perda de massa muscular e consequente diminuição da força; o consumo de cigarro, em que a liberação de radicais livres decorrente do consumo leva à indução de respostas inflamatórias que geram danos celulares e comprometem funçôes metabólicas associadas à massa muscular e força; e a não realização de atividade física, cuja plausibilidade biológica da inter-relação com baixos níveis de força centra-se nos efeitos que deixam de ser gerados por meio das cargas mecânicas provenientes dos movimentos realizados durante a atividade física, que são essenciais para o crescimento e manutenção do sistema músculo esquelético ${ }^{9-11}$.

Outro fator correlato a baixos níveis de força é o status de peso corporal, em que baixo peso pode não estar associado apenas a menor percentual de gordura, mas também relacionado a menor quantidade de massa muscular, o que pode levar a menores escores de força ${ }^{11}$. Por outro lado, indivíduos com excesso de peso são menos ativos fisicamente, o qual pode acarretar menor estímulo da musculatura esquelética, baixa quantidade de massa muscular e níveis de força inferiores em relação aqueles com peso normal ${ }^{11,12}$.

Diante da associação de baixos níveis de força muscular em adolescentes e agravos em saúde, torna-se relevante estimar a prevalência de baixos níveis de força e identificar os fatores correlatos a esta condiçáo, com o objetivo de incentivar ações de prevenção e promoção de saúde para minimizar gastos decorrentes do tratamento desses danos ${ }^{12}$. Porém, não se verificou na literatura estudos com populaçáo brasileira e amostra representativa de adolescentes que explorassem simultaneamente a associação de baixos níveis de força muscular de preensão manual com fatores sociodemográficos e do estilo de vida. Assim, o objetivo do presente estudo foi estimar a prevalência de baixos níveis de FPM e verificar os fatores sociodemográficos, estilo de vida e status de peso corporal associados em adolescentes (14-19 anos) de uma cidade do Sul do Brasil.

\section{Método}

Esta pesquisa epidemiológica de base escolar, com delineamento transversal fez parte do projeto "Guia Brasileiro de avaliação da aptidão física relacionada a saúde e hábitos de vida - etapa 1", o qual foi financiado pelo Conselho Nacional de Desenvolvimento Científico e Tecnológico (CNPq). A pesquisa foi realizada na cidade de São José/SC, na regiáo Sul do Brasil. O município possui Índice de Desenvolvimento Humano de 0,809 , com esperança de vida ao nascer de 77,81 anos, renda per capita de $\mathrm{R} \$ 1.157,43$ e índice GINI de $0,44^{13}$.

\section{População e amostra}

A população desta pesquisa era formada por 5.182 estudantes do ensino médio de escolas públicas estaduais da cidade de São José, distribuídos em 11 escolas elegíveis e 170 turmas do Ensino Médio.

O processo amostral foi determinado em dois estágios: $1^{\circ}$ formado pela estratificação da densidade escolar (tamanho: pequenas, com menos de 200 alunos; médias, com 200 a 499 alunos; e grandes, com 500 estudantes ou mais). No segundo estágio, foi considerado o turno de estudo e a série de ensino. 
Para determinação do tamanho amostral optouse por utilizar o cálculo proposto por Luiz e MAGNANI ${ }^{14}$ para populaçôes finitas, adotando-se nível de confiança de 1,96 (intervalo de confiança de $95 \%$ ), erro tolerável de cinco pontos percentuais, prevalência de $50 \%$ (desfecho não conhecido), e efeito de delineamento de 1,5. Acrescentando-se $20 \%$ para minimizar eventuais perdas e recusas ao estudo e mais $20 \%$ para controle das possíveis variáveis de confusão. A partir desse cálculo seriam necessários 751 adolescentes para compor a amostra. Devido à amostragem por conglomerado, todos os estudantes das turmas foram convidados a participarem da pesquisa, resultou-se em 1.132 alunos com dados coletados. Desse quantitativo, 923 alunos realizaram o teste de força de preensão manual. Os dados utilizados neste trabalho são provenientes da referida pesquisa, em que o cálculo amostral considerou as associaçóes de interesse da investigaçáo que demandassem os maiores números de participantes. Devido a utilização dos dados para testar associaçóes procedeu-se cálculo do poder estatístico disponível para avaliar a associação entre FPM com todas as variáveis de interesse. $\mathrm{O}$ poder estatístico é a probabilidade de deixar de rejeitar uma hipótese nula falsa, ou seja, declarar que não existe uma diferença quando na verdade existe, em que valores apartir de $80 \%$ são considerados adequados para evitar tal erro ${ }^{15}$. Para o presente estudo, a associação entre FPM e as variáveis idade (que obteve poder de $13,0 \%)$, atividade física $(15,0 \%)$, uso de cigarro $(21,9 \%)$, uso de álcool $(29,4 \%)$ e índice de massa corporal $(5,0 \%)$ foram as que apresentaram menor poder estatístico para testes de associação ${ }^{15}$.

\section{Critério de elegibilidade}

Para participar deste estudo, o adolescente deveria ter de 14 a 19 anos e estar regularmente matriculado na rede pública estadual da cidade de Sáo José/SC. Considerou-se como critérios de exclusão a gravidez entre as moças e algum tipo de deficiência que limitasse a realização de algum teste.

\section{Aspectos éticos}

O estudo foi aprovado pelo Comitê de Ética e Pesquisa com Seres Humanos da Universidade Federal de Santa Catarina sob o protocolo CAAE: 33210414.3.0000.0121. Participaram da pesquisa somente os sujeitos que devolveram o Termo de consentimento livre e esclarecido assinado pelos pais ( $<18$ anos) ou por eles mesmos ( $\geq 18$ anos), juntamente com o Termo de assentimento assinado pelo adolescente.

\section{Coleta de dados}

A coleta de dados foi realizada no segundo semestre de 2014, entre os meses de agosto e novembro. O grupo de pesquisadores foi formado por estudantes de graduação e pós-graduação em Educação Física, previamente familiarizados e treinados para aplicação do questionário e testes físicos. O questionário do projeto foi aplicado em sala de aula, sendo que os dados eram de autopreenchimento por parte dos estudantes.

\section{Variável dependente}

Força de preensão manual: para avaliaçáo da força foi utilizado dinamômetro manual da marca Saehan ${ }^{\oplus}$, que possui validação concorrente com dinamômetro Jamar $^{\oplus}(\mathrm{r}=0,976)$ e confiabilidade intra-examinador $(r=0,985)^{1}$. Durante a avaliação, o adolescente ficava em pé com os braços estendidos ao lado do corpo, sem que o equipamento encostasse na coxa. O equipamento ficou localizado entre as falanges distais e a palma da mão, após isso foi solicitado que o adolescente fizesse inspiração e expiração máxima, seguida da maior pressão com a mão no equipamento ${ }^{16}$. O teste foi realizado em ambas as máos de forma alternada, duas vezes, sendo que o melhor resultado de cada mão foi anotado e somado obtendo-se a força total. A FPM foi classificada conforme sexo, nos meninos, aqueles que tiveram FPM menor ou igual a $83 \mathrm{kgf}$ foram classificados com "baixos níveis de força", os que tiveram FPM superior foram classificados como "níveis normais de força" ${ }^{16}$. Nas meninas que tiveram resultados de FPM menor ou igual a $53 \mathrm{kgf}$, foram classificadas com "baixos níveis de força", e aquelas que obtiveram resultado superior foram classificados com "níveis normais de força" de FPM ${ }^{16}$.

\section{Variáveis independentes}

As variáveis sociodemográficas foram: idade em anos completos (categorizada em 14/15 anos, 16/17 anos e 18/19 anos). Esta variável foi categorizada desta forma pelos autores devido a mudanças cognitivas e sociais divergentes entre 
alunos que ingressam no ensino médio (a partir dos $14 / 15$ anos), aqueles que estão na idade adequada (16/17 anos) e alunos que já ultrapassaram os 18 anos e deveriam ter completado o Ensino Médio ${ }^{17}$, pois acabam acumulando outras tarefas extracurriculares, como trabalhar e cuidar da família ${ }^{17}$, reduzindo o nível de atividade física; escolaridade do pai e da mãe, coletada em anos completos (categorizada em até oito anos de estudo e oito anos ou mais de estudo), tal categorização foi a fim de se equiparar com a média de anos de estudo de adultos brasileiros $(7,8 \text { anos })^{18}$; renda familiar coletada conforme Instituto Brasileiro de Geografia e Estatística ${ }^{19}$, em número de salários mínimos recebidos entre os membros da família (salário mínimo equivalente a $\mathrm{R} \$ 724,00$ no período de coleta de dados) (categorizada em até dois salários mínimos (baixa)/de dois a dez salários mínimos (média)/acima de dez salários mínimos (alta).

As variáveis atividade física e uso excessivo de álcool foram avaliadas por questōes da versão Brasileira do Youth Risk Behavior Surveillance (YRBSS), utilizado nos Estados Unidos, traduzido e validado para o Brasil, possuindo índice de concordância moderadamente alta com média $68,3 \%$ e mediana de $68,5 \%{ }^{20}$. As variáveis de uso de cigarro e dieta fazem parte do questionário de "Estilo de vida Fantástico", elaborado pela Canadian Society for Exercise Physiology ${ }^{16}$, traduzido e validado no Brasil, possuindo validação de constructo com capacidade classificatória do instrumento de $75 \%$ e 80,7\%, com um índice Kappa de 0,58 e 0,70, e boa reprodutibilidade com uma alta correlação intraclasse $(\mathrm{R}=0,92 ; \mathrm{p}=0,2)^{21}$.

A atividade física foi coletada pela questão: "Durante os últimos sete dias, em quantos dias você foi ativo fisicamente por pelo menos 60 minutos por dia?", sendo a resposta dicotomizada em pouco ativo (zero a quatro dias) e ativo (cinco dias ou mais), em razão de estudos descreverem que atividade física realizada por pelo menos cinco dias da semana, traz benefícios à saúde do adolescente ${ }^{22}$.

O uso de álcool foi coletado pela questão "Durante os últimos 30 dias, em quantos dias você tomou cinco ou mais doses de bebida alcoólica em uma mesma ocasião? (uma dose corresponde a uma lata de cerveja, uma taça de vinho, uma dose de uísque, pinga, cachaça, rum, vodca e etc.)", aqueles que responderam por pelo menos uma vez, foi considerada resposta positiva (considerado grupo de risco) para consumo de bebida alcoólica, já que o uso de bebida entre os adolescentes está associado à desnutrição, distúrbios mentais e morte por envolvimento em acidentes de trânsito ${ }^{23}$.

O uso de cigarro foi coletado pela pergunta "Você fuma cigarro?", os indivíduos que responderam "Você nunca fumou?" foram considerados saudáveis; e aqueles que responderam mais de 10 por dia; 1 a 10 por dia; nenhum nos últimos seis meses; nenhum no ano passado foram considerados como grupo de risco, pois o uso de cigarro leva a indução de resposta inflamatória, que geram danos celulares e comprometem funções metabólicas associadas a massa muscular e força ${ }^{9}$.

A variável dieta foi coletada pela pergunta: "Você come dieta balanceada?" Considerou-se dieta balanceada: gráos e cereais (5 a 12 porçóes por dia); frutas e vegetais (5 a 10 porçóes por dia); carnes e semelhantes ( 2 a 3 porçôes por dia); leite e derivados ( 3 a 4 porçóes até 16 anos, 2 a 4 porçóes acima de 16 anos) ${ }^{16}$. As variáveis quase nunca; raramente e algumas vezes foram consideradas como resposta pouco frequente (grupo de risco), visto que dieta inadequada é fator de risco para pior perfil metabólico (alta concentração de triglicerídeos e elevada pressão sanguínea), estando associada a baixos níveis de força em adolescentes ${ }^{24}$.

A estatura foi coletada por meio de estadiômetro com tripé da marca Sanny (São Paulo, Brasil) e a massa corporal com balança digital da marca $G$-tech $^{\odot}$ (Zhongshan, China). Foi calculado o IMC a partir da fórmula: IMC = (massa corporal/estatura ${ }^{2}$ ). Para categorizar a variável foram utilizados os pontos de cortes propostos pela Organização Mundial da Saúde ${ }^{25}$, sendo que as categorias baixo peso e peso normal foram agrupadas em peso normal e as categorias sobrepeso e obesidade agrupadas em excesso de peso, pois os estudos indicaram que adolescentes de maior massa corporal apresentam maiores níveis de força de preensão manual ${ }^{11,26}$.

\section{Variável de controle}

A maturação sexual foi autoavaliada pelos adolescentes, utilizando pranchas de desenvolvimento maturacional propostas por MARShall e TANNER ${ }^{27}$. Estas pranchas continham fotografias dos cinco estágios de desenvolvimento maturacional, sendo solicitado que os adolescentes 
observassem com atenção cada fotografia e que marcasse no questionário o que mais se parecia com seu tamanho de órgão genital para os meninos e de tamanho das mamas para as meninas. Os escolares foram orientados individualmente por avaliadores do mesmo sexo sobre objetivo e importância desta avaliação. Como a variável foi inserida no modelo como controle, o seu resultado foi utilizado de modo contínuo.

\section{Análise estatística}

Os dados foram digitados em duplicata no programa Epidata versão 3.1 (Epidata Association, Odense, Dinamarca) a fim de se diminuir algum tipo de erro no momento da digitação, posteriormente ocorreu a checagem automática de consistência e amplitude dentro do próprio programa.

Foi aplicada estatística descritiva e inferencial nos dados, sendo que a normalidade dos dados foi testada com o teste de Shapiro-Wilk para as variáveis contínuas e utilizado o teste de MannWhitney para se verificar as diferenças entre os sexos. Para as associaçóes entre inadequada FPM e as variáveis independentes foi utilizado o teste Qui-quadrado de Pearson. $\mathrm{Na}$ análise bruta e ajustada, foi utilizado teste de Wald e regressão logística binária para estimar as razóes de chances (RC) e intervalos de confiança de 95\%.

A análise de regressão ajustada seguiu modelo hierarquizado, sugerido por VICTORIA et al. ${ }^{28}$, em que no nível distal foram incluídas as variáveis sociodemográficas (idade, escolaridade da mãe e do pai e renda); no nível intermediário as variáveis de estilo de vida (atividade física, uso de cigarro, uso de álcool e dieta); e no nível proximal a variável IMC. Todas as variáveis foram inseridas no modelo independentemente do valor de $p$ na análise bruta.

A análise ajustada hierarquizada adotou procedimento backward, em que inicialmente eram inseridas todas as variáveis do bloco distal e ajustada pelas demais variáveis do mesmo bloco. Em seguida eram inseridas as variáveis do bloco intermediário em conjunto com as do bloco distal e o modelo era ajustado. Por fim, foram inseridas as variáveis do bloco proximal, no qual foi realizado ajuste para outros fatores dos níveis intermediário e distal que ainda permaneciam no modelo. Valor de $p$ abaixo de 0,20 no teste de Wald foi adotado como critério de permanência do fator na análise de regressão. A maturação sexual foi utilizada em todo o modelo independente de seu $p$ valor como variável de controle. Optou-se por fazer análise estratificada por sexo, pois os meninos apresentaram maiores médias de FPM em ambos os braços e consequentemente maior FPM total. Foi utilizado o software Stata 12.0 (StataCorp, College Station, Texas, EUA), com auxílio do comando "svy", devido a amostragem complexa da pesquisa com nível de confiança de $95 \%$.

\section{Resultados}

Ao total foram coletados 923 dados, sendo que a amostra tinha média de 16,1 $(1,1)$ anos de idade, IMC médio $22,2(3,8) \mathrm{kg} / \mathrm{m}^{2}$ e FPM média de 57,9 $(19,2)$ kgf. Os meninos possuíam maior média em relação à idade, massa corporal, estatura e FPM em comparação às meninas $(\mathrm{p}<0,01)$. Não foram encontradas diferenças estatísticas no IMC entre os sexos (TABELA 1).

Mais da metade da amostra era do sexo feminino (54,2\%). A maioria das meninas tinha de 16 a 17 anos $(58,3 \%)$, renda média $(63,9 \%)$ e pais e mães com até oito anos de estudo $(58,3 \%$ e $56,8 \%$, respectivamente). Mais de $80 \%$ das adolescentes eram pouco ativas fisicamente, aproximadamente $15 \%$ faziam uso de cigarro, $35 \%$ uso excessivo de bebida alcoólica e mais de $85 \%$ não tinham dieta balanceada. Com relação ao IMC, 27\% estavam com excesso de peso, para FPM quase $60 \%$ possuíam níveis baixos (TABELA 2).

Nos meninos, a maioria da amostra estava na idade de 16 a 17 anos (56,2\%), os pais e as máes tinham menos de oito anos de escolaridade $(55,5 \%$ e $62,7 \%$, respectivamente) e grande parte das famílias tinha renda média $(73,6 \%)$. A maioria era pouco ativa $(72,4 \%)$ sendo que, $15 \%$ fizeram uso de cigarro, $44 \%$ uso de bebida alcoólica e $83,5 \%$ possuíam dieta equilibrada pouco frequente. Em relação ao IMC um quarto da amostra estava com excesso de peso e a maioria possuía baixos níveis de FPM $(66,3 \%)$ (TABELA 2$)$. 
M: média; DP:

Desvio padrão; ME:

Mediana; IR: Intervalo

Índice de massa

corporal; p: p-valor; 1

Teste T para amostras

independentes; 2 :

Teste Mann-Whitney.
Interquartil; IMC:

TABELA 1 - Valores de média e desvio padrão da idade, variáveis antropométricas e FPM dos braços direito, esquerdo e total dos adolescentes de São José, SC, Brasil

\begin{tabular}{lccccccc}
\hline \multirow{2}{*}{ Variáveis } & \multicolumn{2}{c}{ Total } & \multicolumn{2}{c}{ Masculino } & \multicolumn{2}{c}{ Feminino } & \multirow{2}{*}{ p } \\
\cline { 2 - 7 } & $\mathbf{M} \pm \mathbf{D P}$ & $\mathbf{M E}(\mathbf{I R})$ & $\mathbf{M} \pm \mathbf{D P}$ & $\mathbf{M E}(\mathbf{I R})$ & $\mathbf{M} \pm \mathbf{D P}$ & $\mathbf{M E}(\mathbf{I R})$ & \\
\hline Idade (anos) & $16,1 \pm 1,1$ & $16,0(2,0)$ & $16,2 \pm 1,2$ & $16,0(2)$ & $16,0 \pm 1,1$ & $16,0(2,0)$ & $<0,01^{1}$ \\
Massa (kg) & $61,9 \pm 12,6$ & $60,1(16,0)$ & $65,7 \pm 12,3$ & $64,4(14,1)$ & $58,5 \pm 11,7$ & $55,9(14,0)$ & $<0,01$ \\
Estatura (cm) & $166,4 \pm 8,8$ & $166,2(13,0)$ & $172,7 \pm 7,3$ & $173,1(9,8)$ & $161,3 \pm 6,0$ & $161,0(7,5)$ & $<0,01^{1}$ \\
IMC (kg/m $)$ & $22,2 \pm 3,8$ & $21,4(4,3)$ & $21,9 \pm 3,6$ & $21,5(3,9)$ & $22,43 \pm 4,0$ & $21,5(4,8)$ & $0,31^{2}$ \\
$\begin{array}{l}\text { Máo } \\
\text { direita (kgf) }\end{array}$ & $29,3 \pm 9,6$ & $28,0(14,0)$ & $36,2 \pm 8,8$ & $36,0(12,0)$ & $23,2 \pm 5,2$ & $23,0(6,0)$ & $<0,01^{2}$ \\
$\begin{array}{l}\text { Máo } \\
\text { esquerda (kgf) }\end{array}$ & $28,7 \pm 10,0$ & $26,0(15,0)$ & $35,8 \pm 9,1$ & $36,0(12,0)$ & $22,3 \pm 5,5$ & $22,0(8,0)$ & $<0,01^{2}$ \\
$\begin{array}{l}\text { Força } \\
\text { total (kgf) }\end{array}$ & $57,9 \pm 19,2$ & $54,0(29,0)$ & $71,9 \pm 17,3$ & $72,0(23,0)$ & $45,5 \pm 10,3$ & $45,0(14,0)$ & $<0,01^{2}$ \\
\hline
\end{tabular}

TABELA 2 - Distribuição da amostra e características descritivas de acordo com sexo. São José/SC, Brasil, 2015

IMC: Índice de massa corporal; IC: Intervalo de confiança.

\begin{tabular}{|c|c|c|c|c|}
\hline \multirow{2}{*}{ Variável } & \multirow{2}{*}{$\mathbf{n}$} & Feminino & \multirow[b]{2}{*}{$\mathbf{n}$} & \multirow{2}{*}{$\begin{array}{c}\text { Masculino } \\
\%(\text { IC95\%) } \\
\end{array}$} \\
\hline & & $\%($ IC95\%) & & \\
\hline Total & 613 & $54,2(43,9-64,0)$ & 519 & $45,8(32,9-56,0)$ \\
\hline \multicolumn{5}{|l|}{ Idade (anos) } \\
\hline $14-15$ & 209 & $33,9(30,5-37,3)$ & 152 & $29,3(25,1-33,8)$ \\
\hline $16-17$ & 357 & $58,3(57,3-59,2)$ & 292 & $56,2(52,3-60,0)$ \\
\hline $18-19$ & 47 & $7,8(5,2-11,4)$ & 75 & $14,5(13,9-14,9)$ \\
\hline \multicolumn{5}{|l|}{ Escolaridade da máe } \\
\hline Até oito anos & 347 & $56,8(40,4-71,7)$ & 284 & $55,5(42,6-67,6)$ \\
\hline Oito anos ou mais & 260 & $43,2(28,2-59,5)$ & 227 & $44,5(32,4-57,3)$ \\
\hline \multicolumn{5}{|l|}{ Escolaridade do pai } \\
\hline Até oito anos & 336 & $58,3(48,4-67,5)$ & 318 & $62,7(55,6-69,3)$ \\
\hline Oito anos ou mais & 238 & $41,7(32,4-51,6)$ & 186 & $37,3(30,7-44,3)$ \\
\hline \multicolumn{5}{|l|}{ Renda } \\
\hline Baixa & 167 & $32,7(27,7-38,2)$ & 86 & $20,0(9,0-38,6)$ \\
\hline Média & 325 & $63,9(61,9-65,9)$ & 314 & $73,6(60,2-83,6)$ \\
\hline Alta & 17 & $3,4(0,4-23,3)$ & 27 & $6,4(3,9-10,2)$ \\
\hline \multicolumn{5}{|l|}{ Atividade física } \\
\hline Ativo & 113 & $18,8(16,8-20,8)$ & 139 & $27,6(20,6-36,0)$ \\
\hline Pouco Ativo & 487 & $81,2(79,1-83,2)$ & 364 & $72,4(64,0-79,4)$ \\
\hline \multicolumn{5}{|l|}{ Uso de cigarro } \\
\hline Não & 557 & $85,7(80,2-90,0)$ & 469 & $84,4(74,0-91,1)$ \\
\hline Sim & 47 & $14,3(10,2-19,7)$ & 44 & $15,6(8,7-25,9)$ \\
\hline \multicolumn{5}{|c|}{ Uso de álcool em excesso } \\
\hline Não & 400 & $65,6(61,0-69,8)$ & 346 & $66,8(61,0-72,1)$ \\
\hline Sim & 210 & $34,4(30,1-38,9)$ & 169 & $33,2(27,8-39,0)$ \\
\hline \multicolumn{5}{|l|}{ Dieta adequada } \\
\hline Frequente & 92 & $15,0(13,1-17,0)$ & 85 & $16,5(15,5-17,6)$ \\
\hline Pouco frequente & 519 & $85,0(82,9-86,8)$ & 427 & $83,5(82,4-84,5)$ \\
\hline \multicolumn{5}{|l|}{ IMC } \\
\hline Normal & 388 & $72,9(63,8-80,4)$ & 354 & $74,8(58,0-86,4)$ \\
\hline Excesso de peso & 147 & $27,1(19,6-36,2)$ & 119 & $25,2(13,6-41,9)$ \\
\hline Preensáo manual & \multicolumn{4}{|c|}{$p=\mathbf{0 , 0 6}$} \\
\hline Normal & 200 & $40,3(22,3-61,2)$ & 149 & $33,7(22,0-46,4)$ \\
\hline Baixo & 287 & $59,7(38,7-67,7)$ & 287 & $66,3(53,6-77,0)$ \\
\hline
\end{tabular}


A prevalência de baixos níveis de FPM foi maior nas meninas que praticavam pouca atividade física $(63,1 \%)$, não utilizavam bebida alcoólica $(65,0 \%)$ e tinham IMC normal (65,9\%). Nos meninos aqueles de menor renda $(73,1 \%)$ e que náo consumiam bebida alcoólica em excesso $(68,8 \%)$, possuíam maior prevalência de baixos níveis de FPM (TABELA 3).

$\mathrm{Na}$ análise bruta, meninas pouco ativas fisicamente tiveram maiores chances $(78 \%)$ de terem níveis inadequados de FPM. Àquelas adolescentes que consumiram bebida alcoólica em excesso e que tinham sobrepeso/obesidade apresentaram, respectivamente, $46 \%$ e $62 \%$ de chances a menos de estarem com baixos níveis de FPM. Na análise ajustada, as adolescentes que consumiram bebida alcoólica em excesso e que tinham sobrepeso/obesidade apresentaram, respectivamente, 47\% e 63\% de chances a menos de estarem com baixos níveis de FPM (TABELA 4).

Nos meninos, na análise bruta aqueles de renda alta e que consumiram bebida alcoólica em excesso tinham $66 \%$ e $30 \%$, respectivamente, de chances a menos de terem baixos níveis de FPM. Quando ajustado pelos demais fatores, meninos que as mães estudaram oito anos ou mais, de renda média e alta, tiveram menos chances de terem baixos níveis de FPM (TABELA 5).

TABELA 3 - Prevalência de baixa força de preensão manual de acordo com cada variável independente

\begin{tabular}{|c|c|c|c|c|}
\hline \multirow{2}{*}{ Variável } & \multirow{2}{*}{$\mathbf{n}$} & \multirow{2}{*}{$\begin{array}{c}\text { Feminino } \\
\%(\mathrm{IC} 95 \%) \\
\end{array}$} & \multirow{2}{*}{$\mathbf{n}$} & \multirow{2}{*}{$\begin{array}{c}\text { Masculino } \\
\%(\text { IC95\%) } \\
\end{array}$} \\
\hline & & & & \\
\hline Total & 287 & $59,7(38,7-67,7)$ & & $66,3(53,6-77,0)$ \\
\hline Idade (anos) & & ${ }^{*} p=0,33$ & & ${ }^{*} p=0,11$ \\
\hline 14-15 & 101 & $67,5(50,4-80,9)$ & 92 & $76,0(42,5-93,2)$ \\
\hline $16-17$ & 164 & $56,6(25,4-83,2)$ & 157 & $64,5(58,6-70,1)$ \\
\hline 18-19 & 22 & $54,1(47,2-60,8)$ & 38 & $55,1(39,6-69,6)$ \\
\hline Escolaridade da mãe & & ${ }^{*} p=0,75$ & & ${ }^{*} p=0,05$ \\
\hline Até oito anos & 159 & $58,8(37,9-76,9)$ & 163 & $68,7(58,8-77,2)$ \\
\hline Oito anos ou mais & 125 & $60,1(30,5-84,8)$ & 119 & $63,1(49,4-74,9)$ \\
\hline Escolaridade do pai & & ${ }^{*} p=0,16$ & & ${ }^{*} p=0,16$ \\
\hline Até oito anos & 151 & $56,7(39,2-71,0)$ & 179 & $68,2(44,4-83,1)$ \\
\hline Oito anos ou mais & 119 & $66,1(35,0-87,6)$ & 195 & $60,8(56,4-65,0)$ \\
\hline Renda & & ${ }^{*} p=0,34$ & & ${ }^{*} \mathbf{p}=\mathbf{0 , 0 1}$ \\
\hline Baixa & 83 & $63,6(44,5-79,2)$ & 52 & $73,1(64,4-80,3)$ \\
\hline Média & 147 & $57,7(28,8-82,1)$ & 167 & $63,1(45,1-78,2)$ \\
\hline Alta & 08 & $56,3(24,0-79,2)$ & 10 & $48,4(38,5-58,5)$ \\
\hline Atividade física & & ${ }^{*} \mathbf{p}=\mathbf{0 , 0 3}$ & & ${ }^{*} p=0,21$ \\
\hline Ativo & 45 & $49,0(34,4-63,7)$ & 69 & $57,5(50,7-64,0)$ \\
\hline Pouco ativo & 238 & $63,1(37,5-83,0)$ & 205 & $68,5(46,6-84,4)$ \\
\hline Uso de cigarro & & ${ }^{*} p=0,34$ & & ${ }^{*} p=0,07$ \\
\hline Não & 247 & $61,1(41,7-77,4)$ & 243 & $67,8(55,6-80,0)$ \\
\hline Sim & 38 & $54,1(17,9-86,4)$ & 38 & $58,9(53,2-64,3)$ \\
\hline Uso de álcool em excesso & & ${ }^{*} \mathbf{p}=<\mathbf{0 , 0 1}$ & & ${ }^{*} \mathbf{p}=<\mathbf{0 , 0 1}$ \\
\hline Não & 203 & $65,0(46,8-82,1)$ & 200 & $68,8(55,5-79,5)$ \\
\hline Sim & 83 & $50,2(31,8-68,5)$ & 84 & $60,9(49,4-71,3)$ \\
\hline Dieta adequada & & ${ }^{*} p=0,29$ & & ${ }^{*} p=0,50$ \\
\hline Frequente & 49 & $70,2(46,9-86,3)$ & 44 & $60,5(42,6-75,9)$ \\
\hline Pouco frequente & 237 & $58,0(32,7-79,6)$ & 238 & $67,4(45,9-83,4)$ \\
\hline IMC & & ${ }^{*} \mathbf{p}=\mathbf{0 , 0 2}$ & & ${ }^{*} p=0,18$ \\
\hline Normal & 227 & $65,9(40,5-84,6)$ & 225 & $69,2(50,6-83,1)$ \\
\hline Excesso de peso & 56 & $42,3(30,0-55,7)$ & 62 & $58,3(45,3-70,2)$ \\
\hline
\end{tabular}


RC: Razão de

Chance; IC: Intervalo

de confiança;

*Análise ajustada

pela maturação

sexual e pelas

demais variáveis

independentes

seguindo modelo

hierárquico, aquelas

que tiveram $p \leq 0,20$

foram retiradas;

IMC: Índice de

massa corporal;

números sobrescritos

$(1,2$ e 3$)$ representam

a entrada das

variáveis no modelo

hierárquico para

análise ajustada.
RC: Razão de

Chance; IC: Intervalo de confiança; *

Analise ajustada

pela maturação

sexual e pelas

demais variáveis

independentes

seguindo modelo

hierárquico, aquelas

que tiveram $p \leq 0,20$

foram retiradas; IMC:

índice de massa

corporal; números

sobrescritos (1, 2

e 3) representam

a entrada das

variáveis no modelo

hierárquico para

análise ajustada.
TABELA 4 - Razões de chances e intervalos de confiança de 95\% na associação entre níveis de força inadequada e as variáveis independente da amostra no sexo feminino

\begin{tabular}{|c|c|c|c|c|c|c|}
\hline \multirow{2}{*}{ Variável } & \multirow{2}{*}{ RC } & \multirow{2}{*}{$\begin{array}{c}\text { Análise bruta } \\
\text { (IC95\%) }\end{array}$} & \multirow[b]{2}{*}{$p$} & \multirow{2}{*}{ RC } & \multirow{2}{*}{$\begin{array}{c}\text { Análise ajustada* } \\
\%(\text { IC } 95 \%)\end{array}$} & \multirow[b]{2}{*}{$p$} \\
\hline & & & & & & \\
\hline \multicolumn{7}{|l|}{ Idade (anos) $^{1}$} \\
\hline $14-15$ & 1 & & 0,18 & 1 & & 0,53 \\
\hline $16-17$ & 0,62 & $(0,12-3,25)$ & & 0,72 & $(0,14-3,74)$ & \\
\hline $18-19$ & 0,56 & $(0,30-1,06)$ & & 0,76 & $(0,10-5,67)$ & \\
\hline \multicolumn{7}{|l|}{ Escolaridade da mãe ${ }^{1}$} \\
\hline Até oito anos & 1 & & 0,75 & 1 & & 0,53 \\
\hline Oito anos ou mais & 1,09 & $(0,36-3,31)$ & & 0,81 & $(0,24-2,67)$ & \\
\hline \multicolumn{7}{|l|}{ Escolaridade do pai ${ }^{1}$} \\
\hline Até oito anos & 1 & & 0,16 & 1 & & 0,17 \\
\hline Oito anos ou mais & 1,55 & $(0,64-3,75)$ & & 1,58 & $(0,61-4,10)$ & \\
\hline \multicolumn{7}{|l|}{ Renda $^{1}$} \\
\hline Baixa & 1 & & 0,32 & 1 & & 0,20 \\
\hline Média & 0,78 & $(0,31-1,92)$ & & 0,72 & $(0,29-1,81)$ & \\
\hline Alta & 0,62 & $(0,15-2,52)$ & & 0,53 & $(0,16-1,73)$ & \\
\hline \multicolumn{7}{|l|}{ Atividade física ${ }^{2}$} \\
\hline Ativo & 1 & & 0,03 & 1 & & 0,11 \\
\hline Pouco ativo & 1,78 & $(1,14-2,79)$ & & 1,71 & $(0,73-3,98)$ & \\
\hline \multicolumn{7}{|l|}{ Uso de cigarro $^{2}$} \\
\hline Não & 1 & & 0,35 & 1 & & 0,35 \\
\hline Sim & 0,75 & $(0,27-2,09)$ & & 0,78 & $(0,33-1,87)$ & \\
\hline \multicolumn{7}{|c|}{ Uso de álcool em excesso } \\
\hline Não & 1 & & $<0,01$ & 1 & & $<0,01$ \\
\hline Sim & 0,54 & $(0,46-0,63)$ & & 0,53 & $(0,46-0,63)$ & \\
\hline \multicolumn{7}{|l|}{${\text { Dieta } \text { adequada }^{2}}^{2}$} \\
\hline Frequente & 1 & & 0,29 & 1 & & 0,32 \\
\hline Pouco frequente & 0,58 & $(0,11-3,03)$ & & 0,61 & $(0,11-3,13)$ & \\
\hline \multicolumn{7}{|l|}{$\mathrm{IMC}^{3}$} \\
\hline Normal & 1 & & 0,02 & 1 & & 0,01 \\
\hline Excesso de peso & 0,38 & $(0,19-0,74)$ & & 0,37 & $(0,22-0,66)$ & \\
\hline
\end{tabular}

TABELA 5 - Razões de chances e intervalos de confiança de 95\% na associação entre níveis de força inadequada e as variáveis independente da amostra no sexo masculino

\begin{tabular}{|c|c|c|c|c|c|c|}
\hline \multirow{2}{*}{ Variável } & \multirow{2}{*}{$\mathbf{R C}$} & Análise bruta & \multirow{2}{*}{$p$} & \multirow{2}{*}{$\mathbf{R C}$} & Análise ajustada* & \multirow{2}{*}{$p$} \\
\hline & & (IC95\%) & & & \% (IC95\%) & \\
\hline \multicolumn{7}{|l|}{ Idade (anos) ${ }^{1}$} \\
\hline $14-15$ & 1 & & 0,11 & 1 & & 0,11 \\
\hline $16-17$ & 0,57 & $(0,15-2,08)$ & & 0,59 & $(0,11-2,97)$ & \\
\hline $18-19$ & 0,38 & $(0,08-1,62)$ & & 0,38 & $(0,10-1,48)$ & \\
\hline \multicolumn{7}{|l|}{ Escolaridade da mãe ${ }^{1}$} \\
\hline Até oito anos & 1 & & 0,05 & 1 & & \\
\hline Oito anos ou mais & 0,77 & $(0,59-1,02)$ & & 0,80 & $(0,64-0,99)$ & 0,04 \\
\hline \multicolumn{7}{|l|}{ Escolaridade do pai ${ }^{1}$} \\
\hline Até oito anos & 1 & & 0,16 & 1 & & 0,59 \\
\hline Oito anos ou mais & 0,72 & $(0,37-1,39)$ & & 0,83 & $(0,24-2,88)$ & \\
\hline \multicolumn{7}{|l|}{ Renda $^{1}$} \\
\hline Baixa & 1 & & 0,02 & 1 & & 0,02 \\
\hline Média & 0,63 & $(0,37-1,05)$ & & 0,60 & $(0,41-0,85)$ & \\
\hline Alta & 0,34 & $(0,18-0,63)$ & & 0,36 & $(0,17-0,73)$ & \\
\hline \multicolumn{7}{|l|}{ Atividade física ${ }^{2}$} \\
\hline Ativo & 1 & & 0,21 & 1 & & 0,24 \\
\hline Pouco ativo & 1,60 & $(0,50-5,07)$ & & 1,47 & $(0,53-4,06)$ & \\
\hline
\end{tabular}


TABELA 5 - Razões de chances e intervalos de confiança de 95\% na associação entre níveis de força inadequada e as variáveis independente da amostra no sexo masculino

\begin{tabular}{|c|c|c|c|c|c|c|}
\hline \multirow{2}{*}{ Variável } & \multirow{2}{*}{ RC } & \multirow{2}{*}{$\begin{array}{c}\text { Análise bruta } \\
\text { (IC95\%) }\end{array}$} & \multirow[b]{2}{*}{$p$} & \multirow{2}{*}{$\mathbf{R C}$} & Análise ajustada* & \multirow[b]{2}{*}{$p$} \\
\hline & & & & & $\%(\mathrm{IC} 95 \%)$ & \\
\hline \multicolumn{7}{|l|}{ Uso de cigarro $^{2}$} \\
\hline Não & 1 & & 0,07 & 1 & & 0,58 \\
\hline Sim & 0,67 & $(0,41-1,10)$ & & 0,95 & $(0,70-1,28)$ & \\
\hline \multicolumn{7}{|c|}{ Uso de álcool em excesso $^{2}$} \\
\hline Não & 1 & & $<0,01$ & 1 & & 0,31 \\
\hline $\operatorname{Sim}$ & 0,70 & $(0,64-0,78)$ & & 0,82 & $(0,43-1,55)$ & \\
\hline \multicolumn{7}{|l|}{ Dieta adequada ${ }^{2}$} \\
\hline Frequente & 1 & & 0,50 & 1 & & 0,85 \\
\hline Pouco frequente & 1,34 & $(0,26-6,76)$ & & 1,07 & $(0,22-5,05)$ & \\
\hline \multicolumn{7}{|l|}{$\mathrm{IMC}^{3}$} \\
\hline Normal & 1 & & 0,19 & 1 & & 0,16 \\
\hline Excesso de peso & 0,62 & $(0,21-1,76)$ & & 0,59 & $(0,21-1,68)$ & \\
\hline
\end{tabular}

\section{Discussão}

Os principais achados do estudo foram que a prevalência de baixos níveis de FPM nas meninas foi de $59,7 \%$ e nos meninos de $66,3 \%$. As meninas que não faziam ingestão de bebidas alcoólicas em excesso e com IMC normal tiveram maiores chances de apresentarem baixos níveis de FPM. Os meninos, cujas mães estudaram até oito anos e de renda baixa foram os subgrupos com maiores chances de baixos níveis de força de FPM.

Os resultados verificados no presente estudo em relação à maior prevalência de baixos níveis de FPM nos meninos em relação às meninas foram semelhantes aos achados no estudo de revisão realizado por Tremblay et al. ${ }^{29}$ com estudantes canadenses, em que o predomínio de baixos níveis de FPM, para a faixa etária de 15 a 19 anos foi de $59 \%$ para os meninos e $47 \%$ nas meninas. A possível justificativa para estes achados é o fato de que meninos sáo incentivados desde cedo a realizar atividades físicas, principalmente as coletivas, em que o esforço realizado tende a ser menor em comparação às práticas esportivas individuais, refletindo em menor estresse muscular e, por consequência, menores níveis de força?

Meninas que não faziam uso de bebidas alcóolicas em excesso tiveram maiores chances de apresentarem baixos níveis de FPM. Estudo de SiLva et al. ${ }^{12}$ com adolescentes de 14 a 17 anos da região Oeste de Santa Catarina (Joaçaba), Brasil, também encontrou menor desempenho em teste de força (lombar) em adolescentes que não consumiam bebidas alcoólicas em excesso. O presente achado contradiz a hipótese que indivíduos com comportamentos de risco à saúde apresentam baixos níveis de força. Os achados do presente estudo, que possam indicar relaçáo positiva do consumo excessivo de álcool com níveis adequados de força de preensão manual para meninas, devem ser observados com cautela, sobretudo porque o delineamento do estudo foi transversal e existe a possibilidade de causalidade reversa. Dessa forma, reforça-se a necessidade de os adolescentes aderirem a hábitos saudáveis para promoção da saúde. No presente estudo foi observado o consumo de cinco ou mais doses de bebidas alcóolicas em uma única ocasião (Binge Drinking), caracterizado pelo consumo ocasional de álcool até intoxicação. $\mathrm{O}$ binge drinking está associado a disfunçôes cerebrais durante a codificação verbal, ativação da memória e tarefas de tomada de decisão ${ }^{30}$. Por sua vez, a alternância entre intoxicação e privação de álcool encontrada no binge drinking, parece ter consequências ainda mais deletérias a saúde em crianças e adolescentes que o consumo contínuo de álcool em doses menores e estar associado ao alcoolismo na vida adulta ${ }^{30}$.

Na presente pesquisa foi encontrado que as meninas que tinham IMC normal apresentaram maiores chances de terem baixos níveis de FPM em comparação às que tinham excesso de peso. Estudos publicados previamente demonstraram que adolescentes eutróficas apresentavam menores níveis de FPM em comparação às adolescentes com excesso de peso ${ }^{11,26}$. Sugere-se que o aumento de excesso de peso leva a aumento da massa 
muscular, proveniente do maior esforço da musculatura esquelética no suporte ao excesso de peso adicional, o que pode explicar os maiores níveis de FPM nas meninas com IMC elevado ${ }^{11}$. Além disso, o excesso de peso encontrado nas meninas não necessariamente é referente ao excesso de gordura corporal, pois o indicador antropométrico empregado (IMC) não distingue entre massa de gordura e massa muscular ${ }^{14}$.

Os achados deste estudo indicaram que os escolares do sexo masculino cujas mães apresentavam baixa escolaridade, tinham maiores chances de terem baixos níveis de FPM. No estudo realizado na Espanha com participaçáo de 1.795 estudantes de 12,5 à 18,5 anos de idade, se verificou resultado semelhante ao observado neste estudo, em que baixa escolaridade materna se associou a menores níveis de força muscular em meninas, porém, esta associação não foi verificada nos meninos ${ }^{7}$. Em outro estudo, realizado com participação de 718 escolares de 10 a 18 anos de idade, na cidade de Londrina, Brasil, não se verificou associação entre escolaridade materna e força muscular $^{6}$. Os menores níveis de FPM verificados nos escolares cujas mães tinham menor escolaridade, poderia ser justificado pelo fato de menor escolaridade da mãe estar relacionado a menor conhecimento de aspectos relacionados a saúde e bem-estar, o que pode refletir em falta de informação a respeito de práticas associadas à saúde global, como por exemplo incentivar os filhos a realizarem atividade físicas ${ }^{6}$.

Verificou-se neste estudo que os escolares do sexo masculino que tinham menor renda familiar apresentaram maiores chances de ter baixos níveis de FPM. Os achados desta pesquisa corroboram com os resultados de outros estudos ${ }^{5,8}$. No estudo de JimÉnEZ-PAvón et al. ${ }^{8}$ realizado com adolescentes de 12,5 a 17,5 anos de idade, provenientes de nove países Europeus foi verificado que meninos de baixo nível socioeconômico apresentaram menor força em comparação aos de alto nível socioeconômico. Resultado semelhante foi verificado no estudo de ArTARIA $^{5}$ realizado com residentes da cidade de Java (Malásia) e Cape Town (África do Sul). Esse resultado se justifica, pois baixa renda reflete a ideia de restrito poder aquisitivo e representa componente fundamental no acesso a menor diversidade de atividades no período do lazer, o que denota em diminuição das chances de prática de atividade física e pode refletir negativamente nos escores de força muscular ${ }^{6}$.

Como limitação deste estudo, o delineamento transversal, que impede o estabelecimento de causalidade e temporalidade entre níveis de força muscular e as outras variáveis. Outra limitação desta pesquisa foi a realização da pesquisa em escolas da rede de ensino pública estadual, cujos dados não podem servir de parâmetro para escolas da rede particular de ensino.

A presente pesquisa apresenta contribuição importante para a área da saúde, pois identificou grupos suscetíveis a baixos níveis de força muscular que é um indicador de saúde global. Estes resultados podem auxiliar no desenvolvimento de intervençóes objetivando a diminuição desta condição nestes adolescentes, como aumento da carga horária das aulas de educação física na grade curricular ou incentivo de atividades corporais por meio de projetos extracurriculares em âmbito escolar. Outro aspecto positivo foi o poder da amostra, o qual permite segurança na extrapolação dos dados para a população alvo ${ }^{15}$. Além disso, a utilização da maturação sexual como variável de controle do modelo ajustado objetivando minimizar resultados inexatos em relação aos níveis de preensão manual pode ser considerado como ponto forte desta pesquisa.

Pode-se concluir que três em cinco meninas e, aproximadamente, dois terços dos meninos apresentaram baixos níveis de FPM. Os subgrupos populacionais que apresentaram maiores chances de baixos níveis de FPM foram as adolescentes que eram eutróficas e que não faziam a ingestáo excessiva de bebidas alcóolicas e os adolescentes cujas famílias apresentaram menor renda e cujas mães tinham baixa escolaridade. Contudo, os resultados verificados no presente estudo devem ser interpretados com cautela, haja vista a natureza do delineamento do estudo e a investigaçáo exclusiva de escolares de escolas públicas estaduais.

\section{Abstract}

Prevalence of low levels of muscular strength and associated factors among adolescents in a city in southern Brazil

Muscle strength is required to perform daily activities, and is considered global health marker. The aim of this study was to estimate the prevalence of low handgrip strength levels (HGS) in adolescents and identify 
factors (sociodemographic, lifestyle and weight status) related. The population of schoolchildren in the city of São José, SC, Brazil was formed by 5182 students. For the present school-based study and cross-sectional design, 923 adolescents aged 14 to 19 years were evaluated. HGS was analyzed through handgrip and protocol proposed by the Canadian Society of Exercise Physiology whose cut-off for low power levels is $\leq 53.0 \mathrm{~kg}$ for girls and $\leq 83.0 \mathrm{~kg}$ for boys. The independent variables analyzed were age, paternal and maternal education, family income, physical activity, smoking, food habits and weight status. Student's T-test, Mann-Whitney $\mathrm{U}$ test and binary logistic regression were performed to estimate the odds ratios and confidence interval of $95 \%$. The prevalence of low levels of HGS was $59.7 \%$ for girls and $66.3 \%$ in boys. Girls who did not consume excessive alcoholic beverages and eutrophic and boys whose mothers had low education and low family monthly income were more likely to have low levels of HGS. Efforts to improve levels of HGS should be focused on girls with normal status and boys whose mothers have low education and income. Longitudinal surveys should be proposed to identify predictors of low levels of muscle strength in adolescents.

KeYwords: Muscle Strength Dynamometer; Public Health; Hand Strength; Epidemiology; CrossSectional Studies.

\section{Agradecimentos}

Os autores gostariam de agradecer a Universidade Federal de Santa Catarina, Conselho Nacional de Desenvolvimento Científico $(\mathrm{CNPq})$ e a Coordenação de Aperfeiçoamento de Pessoal de Nível Superior (CAPES) pelo subsídio para esta investigação.

\section{Conflitos de interesse}

Os autores declaram não haver conflito de interesse.

\section{Referências}

1. Reis MM, Arantes PMM. Medida de força de preensão manual - validade e confiabilidade do dinamômetro Saehan. Fisioter Pesqui. 2011;18(2):176-81.

2. Silva DAS, Oliveira AC. Impacto da maturação sexual na força de membros superiores e inferiores em adolescentes. Rev Bras Cineantropom Desempenho Hum. 2010;12(3):144-50.

3. Ortega FB, Silventoinen K, Tynelius P, Rasmussen F. Muscular strength in male adolescents and premature death: cohort study of one million participants. BMJ [Internet]. 2012 [citado 26 jul 2019];345. Disponível em: https://www.bmj.com/ content/345/bmj.e7279.

4. Cohen DD, Gómez-Arbeláez D, Camacho PA, et al. Low muscle strength is associated with metabolic risk factors in Colombian children: the ACFIES study. PloS One. 2014;9(4):e93150.

5. Artaria MD. Socioeconomic and Genetic Factors Influencing the Strength, Weight, Length and Width Measurements of Children. Folia Medica Indonesiana. 2010;46:161-6.

6. Constantino-Coledam DH, Ferraiol PF, de Arruda GA, et al. Associação entre indicadores socioeconômicos com a atividade física e aptidão física relacionada à saúde em adolescentes. Rev Salud Pública. 2013;15:823-36.

7. Jiménez-Pavón D, Ortega FB, Ruiz JR, et al. Influence of socioeconomic factors on fitness and fatness in Spanish adolescents: the AVENA study. Int J Pediatr Obes. 2010;5(6):467-73.

8. Jiménez-Pavón D, Ortega F, Ruiz J, et al. Socioeconomic status influences physical fitness in European adolescents independently of body fat and physical activity: the HELENA study. Nutr Hosp. 2010;25(2):311-6. 
9. Rom O, Kaisari S, Aizenbud D, Reznick AZ. Identification of possible cigarette smoke constituents responsible for muscle catabolism. J Muscle Res Cell Motil. 2012;33(3-4):199-208.

10. Barbosa Filho VC, Silva AL, Bozza R, Rech CR, Campos W. Correlates of Cardiorespiratory and Muscular Fitness among Brazilian Adolescents. Am J Health Behav. 2014;38(1):42-52.

11. Artero EG, España-Romero V, Ortega FB, et al. Health-related fitness in adolescents: underweight, and not only overweight, as an influencing factor. The AVENA study. Scand J Med Sci Sports. 2010;20(3):418-27.

12. Silva DAS, Gonçalves ECA, Grigollo LR, Petroski EL. Fatores associados aos baixos níveis de força lombar em adolescentes do Sul do Brasil. Rev Paul Pediatr. 2014;32(4):360-6.

13. Organização das Naçóes Unidas. O Índice de Desenvolvimento Humano Municipal brasileiro. Brasília: PNUD; Ipea; FJP; 2013.

14. Luiz RR, Magnanini MM. A lógica da determinaçäo do tamanho da amostra em investigaçöes epidemiológicas. Cad Saúde Colet (Rio J). 2000;8(2):9-28.

15. Phillips C. Sample size and power: what is enough? Seminars in Orthodontics. 2002;8(2):67-76.

16. Canadian Society for Exercise Physiology. The Canadian Physical Activity, Fitness and Lifestyle Approach (CPAFLA) CSEP - Health and Fitness Program's Health-Related Appraisal and Counselling Strategy. Ottawa: Canadian Society for Exercise Physiology; 2003.

17. Tavares PA, Souza AP, Ponczek VP. Uma análise dos fatores associados à frequência ao ensino médio na educação de jovens e adultos (EJA) no Brasil. PPE. 2014;44(1):7-35.

18. Brasil. Ministério da Educação. Educação brasileira: indicadores e desafios. Brasília: Fórum Nacional de Educação; 2013.

19. Instituo Brasileiro de Geografia e Estatistica. Metodologia do censo demográfico 2010. Rio de Janeiro: IBGE; 2013.

20. Guedes DP, Lopes CC. Validação da versão brasileira do Youth Risk Behavior Survey 2007. Rev Saúde Pública. 2010;44(5):840-50.

21. Rodriguez Añez CR, Reis RS, Petroski EL. Brazilian version of a lifestyle questionnaire: translation and validation for young adults. Arq Bras Cardiol. 2008;91(2):92-8.

22. Strong WB, Malina RM, Blimkie CJ, et al. Evidence based physical activity for school-age youth. J Pediatr. 2005;146(6):732-7.

23. Australain Goverment. National Health and Medical Research Council. Australian guidelines to reduce health risks from drinking alcohol. Canberra: Commonwealth of Australia; 2009.

24. Moliner-Urdiales D, Ortega FB, Vicente-Rodriguez G, et al. Association of physical activity with muscular strength and fatfree mass in adolescents: the HELENA study. Eur J Appl Physiol. 2010;109(6):1119-27.

25. Onis M, Onyango AW, Borghi E, Siyam A, Nishida C, Siekmann J. Development of a WHO growth reference for schoolaged children and adolescents. Bull World Health Organ. 2007;85(9):660-7.

26. Leena P, Mardiikar P, Varsha S. Physical fitness parameters and their correlation with bmi in children and adolescents. JARBS. 2014;6:106-11.

27. Marshall WA, Tanner JM. Variations in pattern of pubertal changes in girls. Arch Dis Child. 1969;44:291-303

28. Victora CG, Huttly SR, Fuchs SC, Olinto MTA. The role of conceptual frameworks in epidemiological analysis: a hierarchical approach. Int J Epidemiol. 1997;26(1):224-7.

29. Tremblay MS, Shields M, Laviolette M, Craig CL, Janssen I, Connor Gorber S. Fitness of Canadian children and youth: results from the 2007-2009 Canadian Health Measures Survey. Health Rep. 2010;21(1)1:7-20.

30. Petit G, Maurage P, Kornreich C, Verbanck P, Campanella S. Binge drinking in adolescents: a review of neurophysiological and neuroimaging research. Alcohol Alcohol. 2014;49(2):198-206.

ENDEREÇO

Tiago Rodrigues de Lima

Rua Professor Clementino de Britto, 362, ap. 334

88070-150 - Capoeiras

Florianópolis - SC - BRASIL

e-mail: tiagopersonaltrainer@gmail.com
Recebido para publicação: 03/12/2015

Revisão: 16/o8/2017

Aceito: 18/09/2017 